Working Paper 99-05

Economics Series 03

February 1999
Departamento de Economía Universidad Carlos III de Madrid

Calle Madrid, 126

28903 Getafe (Spain)

Fax (341) 624-98-75

\title{
MULTUEVEL INTERTERRITORIAL CONVERGENCE AND ADDITIVE MULTIDIMENSIONAL INEQUALITY DECOMPOSITION
}

\author{
Rafael Salas *
}

\begin{abstract}
In this paper we justify the use of the between-groups S-convex relative inequality to measure interregional convergence, within an axiomatic normative context. In addition, the use of additive decomposable inequality indices makes the additive multidimensional (and multilevel) inequality decomposition possible, and help us better understand the evolution of the between- and the withingroups inequality terms (and convergence) when the number of regions increases. We have detected a weak interterritorial divergence process in Spain in the period 1980-1995 using the Eurostat per capita GDP data at all levels, although it is mainly generated at the broad NUTS 1 areas, which is compatible with high level of increasing polarization at this level.
\end{abstract}

Keywords: Regional Convergence, Multidimensional Inequality Decomposition

* Salas, Departamento de Economía, Universidad Carlos III de Madrid. E-mail: salas@eco.uc3m.es

This project has benefited from the financial support of FIES. 


\section{Multilevel Interterritorial Convergence and Additive Multidimensional Inequality Decomposition}

\section{Introduction}

The purpose of this paper is twofold. First, interterritorial economic convergence is defined in terms of the reduction in the classical between-regions' inequality. Classical normative assumptions on inequality, such as symmetry or S-convexity, will prove useful in analyzing interterritorial convergence. The $\beta$ - and $\sigma$-convergence measures first suggested in the economic growth literature by Barro and Sala-i-Martin $(1990,1992)$ fail to meet such normative properties.

Second, by using specific additive decomposable measures we are able to deal satisfactorily with multilevel interterritorial convergence decomposition. More generally, it can be proved that the use of these additive decomposable inequality indices produces an appropriate multidimensional inequality decomposition. As a particular case, Cowell (1985) detected that this property is satisfied by the classical Theil index. The generalization here allows for any general entropy index, including the appealing Theil 0 population-weights-based decomposable measure. Wodon (1998) has proposed an analogous multidimensional inequality decomposition for the Gini coefficient. We find that our methodology has some advantages. We explain the meaning of the difference of the between-groups component better when the number of groups becomes larger, as a second-order between-groups component. This difference is independent of the order followed in the disaggregation process and does not depend on the within-groups individual data. We contribute to a better understanding of the evolution of the between- and within-groups inequality terms, as the number of groups increases; and therefore to the evolution of the convergence process at different territorial levels. Finally, using the Eurostat 1980-1995 per capita GDP data set, we empirically apply our ideas to the Spanish territorial units. 
The paper is organized as follows. In the next section the main definitions, axioms and properties are presented. In section 3, the empirical analysis is carried out and finally, in section 4 , the main conclusions are summarized.

2. Definitions, axioms and propositions

Let $N_{t}=\left\{1,2, \ldots, n_{t}\right\}$ be a set with $n_{t}$ individuals as the population at period t. Consider a disjoint exhaustive partition of the whole population into $\mathrm{K}$ subgroups (such as $\mathrm{K}$ regions),

$N_{t}^{* K}=\left\{N_{t}^{1}, N_{t}^{2}, \ldots, N_{t}^{K}\right\}$

where

$N_{t}^{k}=\left\{1,2, . ., n_{t}^{k}\right\}$

is a $n^{k}$-person subset of $N_{t}$, with

$\sum_{k=1}^{K} n_{t}^{k}=n_{t}$

Let

$Y_{t}=\left\{Y_{1, t}, Y_{2, t}, \ldots, Y_{n_{t},}\right\}$

be the set of non-negative real income levels of the population at period $t$ and

$Y_{1}^{k}=\left\{Y_{1, l}^{k}, Y_{2, l}^{k}, \ldots, Y_{n_{t}^{k}, t}^{k}\right\}$

be the set of income levels of the population in region $\mathrm{k}$ containing $\mathrm{n}^{\mathrm{k}}$, persons at period $\mathrm{t}$, and be

$\bar{Y}_{t}=\sum_{j=1}^{n_{t}} Y_{j, l} / n_{t}$

and 
$\bar{Y}_{t}^{k}=\sum_{j=1}^{n_{t}^{k}} Y_{j, l}^{k} / n_{t}^{k}$

the whole population per capita income and the region $\mathrm{k}$ per capita income, respectively.

Let us define the interregional convergence among the $K$ regions between $t$ and $t+h$ as the change in the between-groups inequality index produced in the period:

$C_{t, t+h}^{K}=I_{t+h}^{B, K}\left(\bar{Y}_{t+h}^{1}, \ldots, \bar{Y}_{t+h}^{K} ; n_{t+h}^{1}, \ldots, n_{t+h}^{K}\right)-I_{t}^{B, K}\left(\bar{Y}_{t}^{1}, \ldots, \bar{Y}_{t}^{K} ; n_{t}^{1}, \ldots, n_{t}^{K}\right)$

The between-groups inequality index is defined classically as the inequality index, $\mathrm{I}: \mathfrak{R}^{\mathrm{nt}}{ }_{++} \rightarrow \mathfrak{R}$, computed from a distribution in which all individuals are assigned their own subgroup mean income:

$I_{t}^{B, K}\left(\bar{Y}_{t}^{1}, \ldots, \bar{Y}_{t}^{K} ; n_{t}^{1}, \ldots, n_{t}^{K}\right)=I_{t}\left(\bar{Y}_{t}^{1} \Phi\left(n_{t}^{1}\right), \ldots, \vec{Y}_{t}^{K} \Phi\left(n_{t}^{K}\right)\right)$

Where $\Phi\left(\mathrm{n}_{\mathrm{t}}\right)=(1, \ldots, 1) \in \mathfrak{R}^{\mathrm{nt}}{ }_{+}$is the transposed $\mathrm{n}_{\mathrm{t}}$-dimensional vector consisting of ones. Assume that the inequality index is temporally invariant, then:

$I_{t}\left(\bar{Y}_{t}^{1} \Phi\left(n_{t}^{1}\right), \ldots, \vec{Y}_{t}^{K} \Phi\left(n_{t}^{K}\right)\right)=I\left(\bar{Y}_{t}^{1} \Phi\left(n_{t}^{1}\right), \ldots, \vec{Y}_{t}^{K} \Phi\left(n_{t}^{K}\right)\right)$

Let us impose the following classical assumptions on the inequality index and analyze their normative implications for the measure of proposed interterritorial convergence.

\section{Al Symmetry}

The inequality index $\mathrm{I}: \mathfrak{R}^{\mathrm{nt}}{ }_{++} \rightarrow \mathfrak{R}$ is symmetric in $\mathrm{Y}$, that is $\mathrm{I}(\mathrm{Y})=\mathrm{I}(\mathrm{PY}), \mathrm{P}$ being any permutation matrix. This imposes a restriction on the proposed convergence measure. Territorial convergence measures do not change under any permutation on the territorial mean income levels between two regions with the same population. The $\beta$-convergence measure proposed by Barro and Sala-i-Martin $(1990,1992)$ does not satisfy this anonymity 
axiom and makes it very much dependent on the rankings of the initial distribution. For example, if there are two regions each with two people and real regional mean incomes represented by $(100,100 ; 200,200)$ and in the next period the positions are reversed to $(200$, $200 ; 100,100), \beta$-convergence will compute a positive convergence value. However, our index will reveal a zero net convergence process (as if there is at first a process of convergence and then a counteracting divergence process of the same magnitude).

\section{A2 Non-negativity}

The inequality index is non-negative and equals zero if and only if perfect equality holds. This technical assumption on inequality guarantees a zero convergence value under changes along perfect regional equality.

\section{A3 Differentiability}

The inequality index is twice continuously differentiable, and so becomes the convergence proposed index.

\section{A4 S-convexity}

The inequality index $I(\cdot)$ is strictly $S$-convex. This is equivalent to $I(Y)>I(A Y)$, where $A$ is any bistochastic matrix that is not a permutation matrix. This is also equivalent to requiring that the inequality index satisfy the principle of transfers: a transfer from a richer to poorer person, with no reranking between them, reduces inequality. This axiom makes our convergence measure consistent with the Lorenz domination criterion when the mean income of the distribution does not change. In this case, the measure is also consistent with any Sconcave social evaluation function W(Y), Atkinson (1970) and Dasgupta et al. (1973). Neither the $\beta$ - nor the $\sigma$-convergence measures are S-convex. Consider the previous example with two regions each with two people and mean incomes represented by $(100,100 ; 200,200)$. If in the next period the positions are $(250,250 ; 50,50), \beta$-convergence will compute a positive(!) convergence value, while our S-convex index will compute a negative 
convergence value. The $\sigma$-convergence (standard deviation of logs) measure is not S-convex at the extreme tails of the distribution, Creedy (1977).

A5 The population replication axiom (PRA)

The inequality index satisfies the $P R A$, that is $I(Y)=I(Y, Y, \ldots, Y)$, where $(Y, Y, \ldots, Y) \in \Re^{\text {mt }}{ }_{++}$ given any positive integer $r$. If we replicate the population $r$ times, the inequality remains unchanged. This is a very common assumption when comparing income distributions that differ in sample size, Dasgupta et al. (1973) and Shorrocks (1983). This axiom together with A4 makes our convergence measure consistent with the Lorenz domination criterion when overall mean income does not change but population changes, and with any S-concave social evaluation function that satisfies the PRA.

A6 Income-homogeneity of degree zero

The inequality index is a relative index (homogeneous of degree zero in income levels), that is, $\mathrm{I}(\mathrm{Y})=\mathrm{I}(\mathrm{kY})$, for any real scalar $\mathrm{k}>0$. This axiom together with $\mathrm{A} 4$ and $\mathrm{A} 5$ makes our convergence measure consistent with the Lorenz domination criterion when overall mean income and population change, and with any S-concave ray-increasing social evaluation function that satisfies the PRA, see Shorrocks (1983). Both $\beta$ - and $\sigma$-convergence measures satisfy $\mathrm{A} 6$ as they are intended to measure reductions in relative regional disparities.

\section{A7 Additive inequality decomposability}

The inequality index is additive decomposable if it can be written as (Shorrocks, 1980):

$$
\begin{aligned}
& I\left(Y_{1, l}, Y_{2, l}, \ldots, Y_{n, t}\right)=I\left(\bar{Y}_{t}^{1} \Phi\left(n_{t}^{1}\right), \ldots, \bar{Y}_{t}^{K} \Phi\left(n_{t}^{K}\right)\right)+\sum_{k=1}^{K} w_{t}^{k}\left(\bar{Y}_{t}^{1}, n_{t}^{1}, \ldots, \bar{Y}_{t}^{K}, n_{t}^{K}\right) I\left(Y_{t}^{k}\right)= \\
& I^{B, K}+I^{W, K}
\end{aligned}
$$

The two terms of the right-hand side expression are the between-groups and within-groups inequality components, respectively. 
It is well known that $A 7$ imposes several restrictions on the class of inequality measures considered. Shorrocks (1980) considered A7 together with A1, A2, A3, A5 and A6 and proved that the inequality indices must adopt the form of the General Entropy class, which are strictly S-convex and therefore satisfy $\mathrm{A} 4$, the principle of transfers. This class has the property that the weights take the form:

$$
w_{t}^{k}(.)=\frac{n_{t}^{k}}{n_{t}}\left(\frac{\bar{Y}_{t}^{k}}{\bar{Y}_{l}}\right)^{c}
$$

A8 Additive multilevel inequality decomposability

The inequality index is additive multilevel decomposable. If we make a subpartition $\mathrm{S}$ from partition $\mathrm{K}$ (such as the $\mathrm{S}$ subregions within the $\mathrm{K}$ regions), then the inequality index can be written as:

$$
\begin{aligned}
& I\left(Y_{1, t}, Y_{2, \imath}, \ldots, Y_{n, t}\right)=I\left(\bar{Y}_{\imath}^{1} \Phi\left(n_{t}^{1}\right), \ldots, \vec{Y}_{\imath}^{K} \Phi\left(n_{t}^{K}\right)\right)+\sum_{k=1}^{K} w_{t}^{k}(\cdot) I\left(\bar{Y}_{t}^{k 1}, \ldots, \bar{Y}_{t}^{k S k}\right)+\sum_{s=1}^{S} w_{t}^{s}(\cdot) I\left(Y_{t}^{s}\right) \\
& I^{B, K}+I^{B, S K}+I^{W, S}
\end{aligned}
$$

where $S_{k}$ is the number of subregions within region $k$.

We shall follow Wodon (1998) and call the new second term of the right-hand side expression, $\mathrm{I}^{\mathrm{B}, \mathrm{SK}}$, the second-order between-groups inequality along the subpartition $\mathrm{S}$ within the groups of the first partition $\mathrm{K}$. It is obtained as a weighted sum of the between-groups inequality along the subpartition $\mathrm{S}$ within any group of the first partition $\mathrm{K}$. The weighting scheme is the same as the within-groups component weights.

The overall inequality can then be divided into the following three components: (1) a firstorder between-groups inequality along the first partition, plus (2) a second-order betweengroups inequality along the second partition within the groups of the first partition, plus (3) a within-groups component for the groups of the second partition. Similarly, the overall convergence along the second partition can be divided into two components: (1) the convergence along the first partition plus (2) a second-order convergence of the second 
partition along the first partition. This procedure can be generalized to any finite multilevel decomposition in subpartitions.

As well, if the inequality index is additive decomposable, then we obtain

$$
I\left(Y_{1, l}, Y_{2,}, \ldots, Y_{n,}\right)=I^{B, K}+I^{W, K}=I^{B, S}+I^{W, S}=I^{B, K}+I^{B, S K}+I^{W, S}
$$

which has the property that

$$
I^{B, S K}=I^{B, S}-I^{B, K} \geq 0
$$

First, the second-order between-groups inequality can be computed as the difference between the two between-groups of the two partitions. Second, it is not negative, indicating that the larger the number of groups, the larger the between-groups component. Naturally, the between-groups component converges to the overall inequality when the number of groups approaches the number of individuals. Note also that it increases exactly the same amount as the within-groups component decreases as

$$
I^{B, S K}=I^{W, S}-I^{W, K} \geq 0
$$

\section{Proposition 1}

If the inequality index satisfies $\mathrm{A} 1, \mathrm{~A} 2, \mathrm{~A} 3, \mathrm{~A} 5, \mathrm{~A} 6$ and $\mathrm{A} 7$, then it also satisfies $\mathrm{A} 8$. [Recall that $\mathrm{A} 4$ is also satisfied provided that the inequality index belongs to the General Entropy class, Shorrocks (1980)].

The proof of this proposition is shown in a separate section.

We can generalize this theorem highlighting more properties of the proposed decomposition. 
A9 Additive multidimensional inequality decomposability

The inequality index is additive multidimensional decomposable. If we make any two disjoint exhaustive partitions of the entire population $\mathrm{N}^{*}{ }_{\mathrm{t}}$ and $\mathrm{N}^{* \mathrm{~J}}$ (say two subgroups partitioned along two dimensions such as regions and education level), then we obtain the subpartition $\mathrm{N}^{* \mathrm{KJ}}$ formed with the set of all the mutually exclusive $\mathrm{N}^{*}{ }_{\mathrm{t}} \mathrm{x} \mathrm{N}^{* J}{ }_{\mathrm{t}}$ subgroups,

$N_{t}^{* K} \times N_{t}^{* J}=\left\{N_{t}^{11}, N_{t}^{12}, \ldots, N_{t}^{K J}\right\}$

where

$N_{t}^{k j}=\left\{1,2, \ldots, n_{t}^{k j}\right\}$

is a $\mathrm{n}^{\mathrm{kj}}{ }_{\mathrm{t}}$-persons subset $\mathrm{N}_{\mathrm{t}}^{\mathrm{k}} \cap \mathrm{N}_{\mathrm{t}}^{\mathrm{j}}$ of $\mathrm{N}_{\mathrm{t}}$, with

$\sum_{k=1}^{K} \sum_{j=1}^{J} n_{t}^{k j}=n_{t}$

Let us rename the subpartition $\mathrm{N}^{* \mathrm{KJ}}$ as the subpartition $\mathrm{N}^{* \mathrm{P}}$

$N^{* P}=N_{t}^{* K} \times N_{t}^{* J}=\left\{N_{1}^{1}, N_{t}^{2}, \ldots, N_{t}^{P}\right\}$

where

$N_{\imath}^{p}=\left\{1,2, \ldots, n_{t}^{p}\right\}$

is a $n^{\mathrm{p}}{ }_{\mathrm{t}}$-persons subset $\mathrm{N}_{\mathrm{t}}^{\mathrm{p}}=\mathrm{N}^{\mathrm{k}}{ }_{\mathrm{t}} \cap \mathrm{N}_{\mathrm{t}}^{\mathrm{j}}$ of $\mathrm{N}_{\mathrm{t}}$, with

$\sum_{p=1}^{P} n_{t}^{p}=n_{t}$

Then the inequality index can be written in the following ways, depending on the sequence followed to construct the subpartitions $\mathrm{N}^{* \mathrm{KJ}}$ or $\mathrm{N}^{* \mathrm{~K}}$, which are eventually identical: 


$$
\begin{aligned}
& I\left(Y_{1, l}, Y_{2, l}, \ldots, Y_{n, t}\right)=I\left(\bar{Y}_{t}^{1} \Phi\left(n_{t}^{1}\right), \ldots, \bar{Y}_{t}^{K} \Phi\left(n_{t}^{K}\right)\right)+\sum_{k=1}^{K} w_{t}^{k}(\cdot) I\left(\bar{Y}_{t}^{k 1}, \ldots, \bar{Y}_{t}^{k J}\right)+\sum_{p=1}^{P} w_{t}^{p}(\cdot) I\left(Y_{t}^{p}\right) \\
& I^{B, K}+I^{B, J K}+I^{W, P} \\
& I\left(Y_{1, l}, Y_{2, t}, \ldots, Y_{n, t}\right)=I\left(\bar{Y}_{t}^{1} \Phi\left(n_{t}^{1}\right), \ldots, \bar{Y}_{t}^{J} \Phi\left(n_{t}^{J}\right)\right)+\sum_{j=1}^{J} w_{t}^{j}(\cdot) I\left(\bar{Y}_{t}^{j 1}, \ldots, \bar{Y}_{t}^{j K}\right)+\sum_{p=1}^{P} w_{t}^{p}(\cdot) I\left(Y_{t}^{p}\right) \\
& I^{B, J}+I^{B, K J}+I^{W, P}
\end{aligned}
$$

In this case, $\mathrm{I}^{\mathrm{B}, \mathrm{KK}}$ stands for the second-order between-groups inequality along partition $\mathrm{J}$ within the groups of partition $\mathrm{K}$ (and being $\mathrm{I}^{\mathrm{B}, \mathrm{KJ}}$, the opposite). It is obtained as a weighted sum of the between-groups inequality along the partition $\mathrm{J}$ within any group of the partition $\mathrm{K}$.

Again if the inequality index is additive decomposable, then we obtain

$$
\begin{aligned}
& I\left(Y_{1, l}, Y_{2, l}, \ldots, Y_{n, f}\right)=I^{B, K}+I^{W, K}=I^{B, J}+I^{W, J}=I^{B, P}+I^{W, P}= \\
& I^{B, K}+I^{B, X J}+I^{W, P}=I^{B, J}+I^{B, J K}+I^{W, P}
\end{aligned}
$$

which has the properties that

$$
\begin{aligned}
& I^{B, P}=I^{B, K}+I^{B, K J}=I^{B, J}+I^{B, J K} \geq 0 \\
& I^{W, P}=I^{W, K}+I^{B, K J}=I^{W, J}+I^{B, J K} \geq 0
\end{aligned}
$$

These are similar to the ones described in connection with A8. As well, the overall betweenor within-groups components of the mutually exclusive subgroups are generated independently of the order followed in the decomposition sequence. This property is not met by the Gini coefficient, see Wodon (1998). Again, the larger the groups, the larger/lower the between-/within-groups component, tending to the value of the overall inequality/zero as disaggregation approaches individuals as partitions. We think these properties should be required to give good explanations for the shifts in inequality when analyzing multilevel or multidimensional inequality decomposition. Cowell (1985) noted that the classical Theil index satisfied this axiom. A generalization for the whole General Entropy class follows.

\section{Proposition 2}

If the inequality index satisfies $\mathrm{A} 1, \mathrm{~A} 2, \mathrm{~A} 3, \mathrm{~A} 5, \mathrm{~A} 6$ and $\mathrm{A} 7$, then it also satisfies $\mathrm{A} 9$. 
3. The empirical results

We apply our methodology to the interterritorial convergence in Spain at different levels and make use of two sources of regional data. The first is the longer biannual BBV Regional per capita GDP data set from 1955 to 1991 and the second is the recent shorter annual Eurostat per capita GDP series from 1980 to 1995 . Both series contain data for the Autonomous Community (Spanish regions) and provincial (Spanish subregions) levels, so we can take advantage of our methodology.

First, Graph 1 shows the well known result, reported elsewhere ${ }^{1}$, that positive per capita regional GDP convergence is produced from 1955 to 1980 . From then on, this process is decelerated. Moreover, we can also assess that this convergence process is even greater at the provincial level. This is due to the second-order convergence process produced among provinces within the Autonomous Communities (AC) from 1955 to 1983 (with an exception in 1975), as is observed in Graph 2. Again this convergence process is not so clear from 1983 onwards.

Nevertheless, the recent picture is clearer from analyzing the Eurostat data set, where the data is presented at the NUTS1 (big areas), NUTS2 (AC) and NUTS3 (provinces) level. According to this data set, the convergence process is (weakly) reversed from 1980 onwards, as shown in Graph 3. Moreover, the divergence process is mainly generated at the NUTS 1 level. No second-order divergence, neither among the AC within the NUTS1, nor among the provinces within the $\mathrm{AC}$, is observed in the period as shown in Graph 4.

This divergence process in the period seems not to be dependent on the particular proposed convergence index used. Graph 5 shows the same results for the Theil 0 index. The reason is that the between-groups S-convex relative inequality is clearly reduced at all levels. In Graph 6 , relative dispersion of the NUTS1 values across time is presented. It reveals clearly that relative dispersion has increased in the period. This seems to be very much the same at the provincial level. Graph 7 shows the population-weighted Lorenz curves differences from both the period 1980-1992 and from the period 1980-1995. In the period 1980-1992 we obtain a 
strict negative Lorenz dominance result, and in the whole period 1980-1995 we obtain an almost negative Lorenz dominance result, except from the very lowest tail of the distribution.

These empirical facts are not well captured by either the non-weighted $\beta$ - or the non-weighted $\sigma$ - convergence ${ }^{2}$. We would like to point out two important facts in relation to the comparison with these alternative convergence measures.

First of all, the non-anonymous non-S-convex and non-weighted $\beta$-convergence measure gives a non-significant negative convergence process at the NUTS1 level in the whole period (Graph 10). But it reports a significant positive convergence (!), at the $92 \%$ level, at the provincial level (Graph 12), when there is almost a negative Lorenz curve dominance in the period. This result is partly due to the dependence of the measure on the initial ranking (and therefore to the violation of the S-convexity condition) and is also partly because the index is not weighted for population.

Let us stress this second characteristic: the importance of the population-subgroups-size weighting scheme in the convergence measurement. In Graph 8 we present the same Lorenz curves without the population weights. Notice the great difference from Graph 7. The misleading result may, in part, come from the fact that Lorenz dominance is not produced in this case. Real S-convex indices will not necessarily capture the true result based on nonweighted population data sets. Graph 9 shows how the divergence process, coming from nonpopulation-weighted Theil 1 indices, is less important in this period than when it is measured by the appropriate population-weighted indices.

Finally, we point out another relevant result that arises from the previous evidence. A great deal of spatial polarization (at NUTS1 level) has been observed in this period. The fact that the divergence is mainly produced at the NUTS1 level, but not at the intermediate AC (nor at the between-provinces) level within NUTS1 as shown in Graph 4, gives some intuition on the increasing polarization. The information in Graphs 6,10 and 11 gives more intuition on this

\footnotetext{
' See, for instance, Raymond and García (1994), Mas et al. (1994), De la Fuente (1996) and Rabadán and Salas (1996).

${ }^{2}$ The non-weighted $\beta$ - or the non-weighted $\sigma$-convergence measures do not explicitly take into account the population size of the subgroups (they are computed as if all subgroups have the same population size), and they have nothing to do with the between-groups weighted sum of the previous section.
} 
NUTS1 polarization between two groups: Noreste, Este and Madrid, on the one hand; and the rest, on the other. In Graph 11, three subperiods are taken into account (1980-85; 1985-90; 1990-95) for the specific NUTS1. We can anticipate that a great deal of conditional convergence is taking place. If specific NUTS1 dummy variables were considered in the convergence equation, a significant negative beta parameter will emerge. Some authors interpret this fact as indicating that conditional convergence toward the particular NUTS1 steady states is being produced. Moreover, the marked separation of the cases into two different sets of NUTS1, in Graphs 10 and 11, suggests that a great deal of polarization is taking place. This needs to be explored in more detail in future research.

\section{Conclusions}

In this paper we justify the use of a between-groups S-convex relative inequality to measure interregional convergence, within an axiomatic normative context. We have detected a weakly divergent interterritorial process in the period 1980-1995 using the Eurostat per capita GDP data. This divergence was not fully detected by either the traditional non-weighted $\beta$ - or the non-weighted $\sigma$-convergence measures.

In addition, the use of additive decomposable inequality indices makes the additive multidimensional (and multilevel) inequality decomposition possible and helps us better understand the evolution of the between- and within-group inequality terms (and convergence) when the number of regions increases. We observe that this divergence is mainly generated at the NUTS1 level, which is compatible with the high level of increasing polarization observed at this level. 

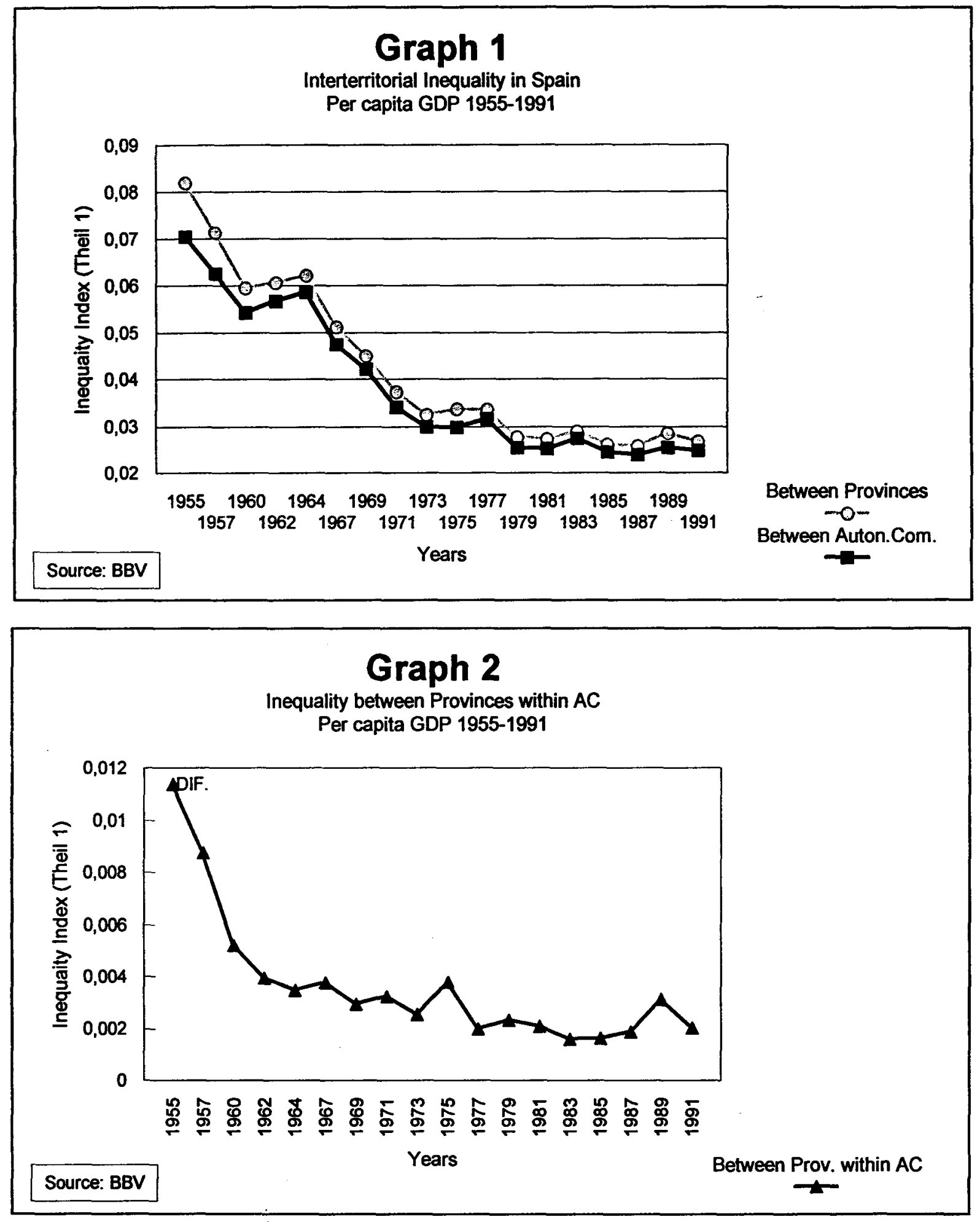

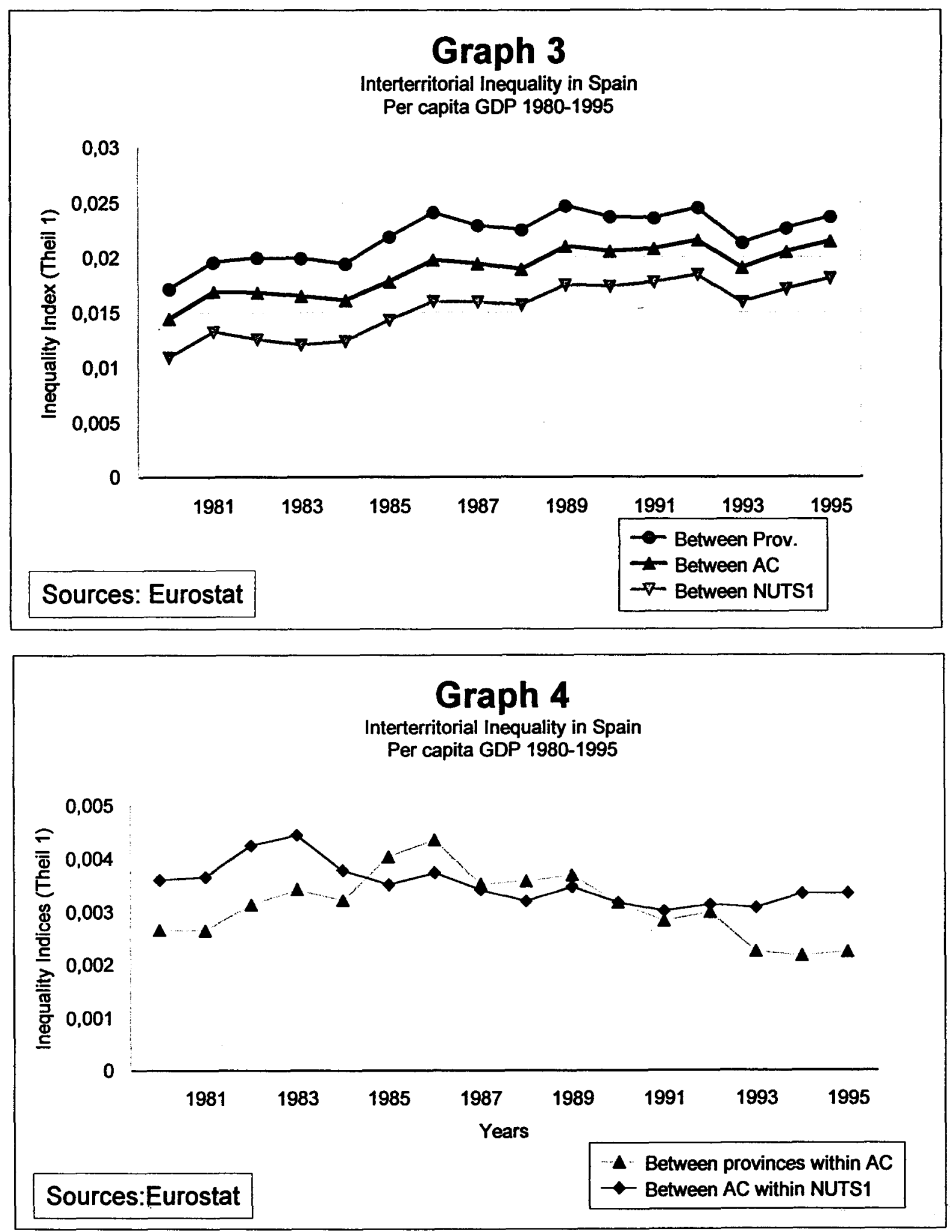

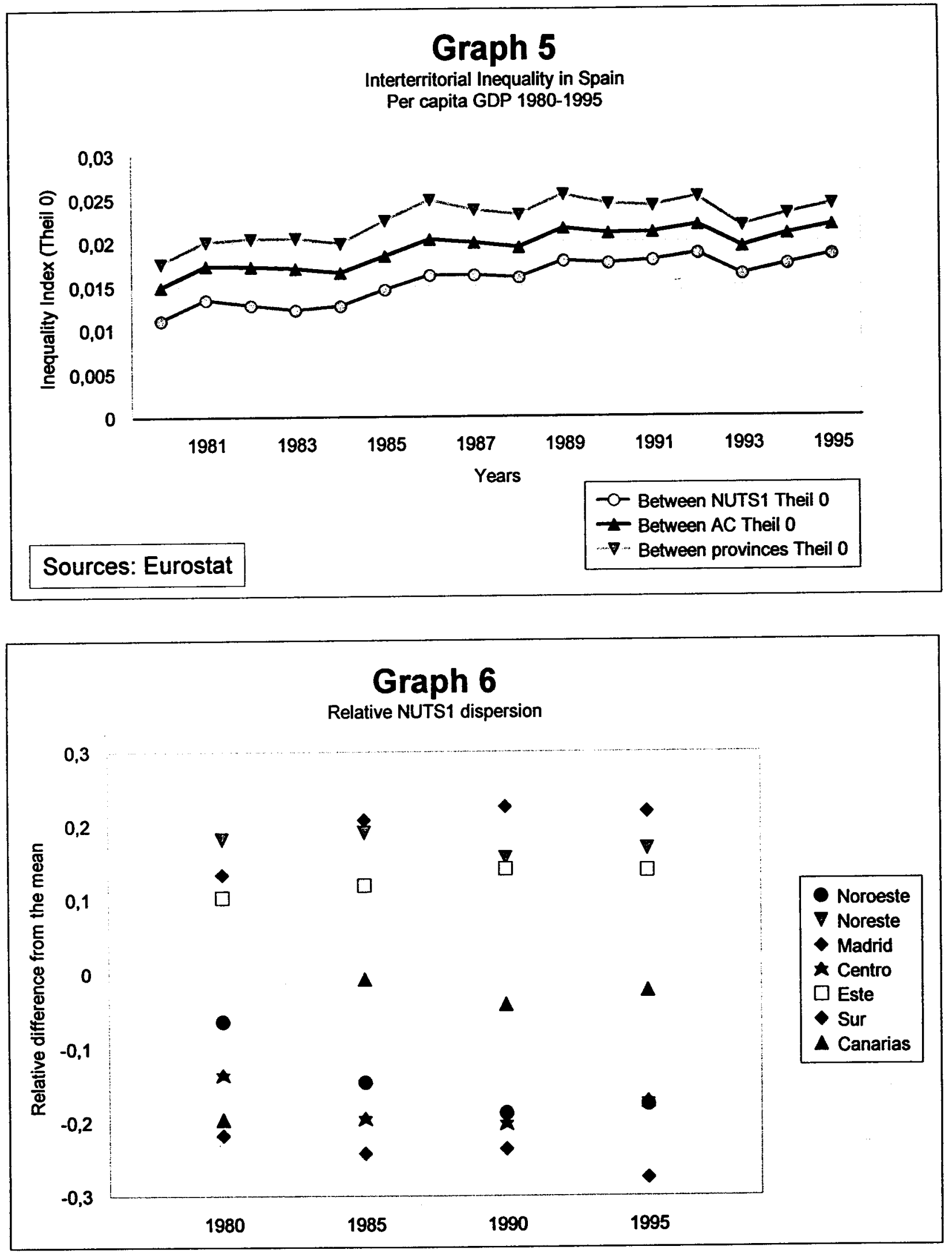


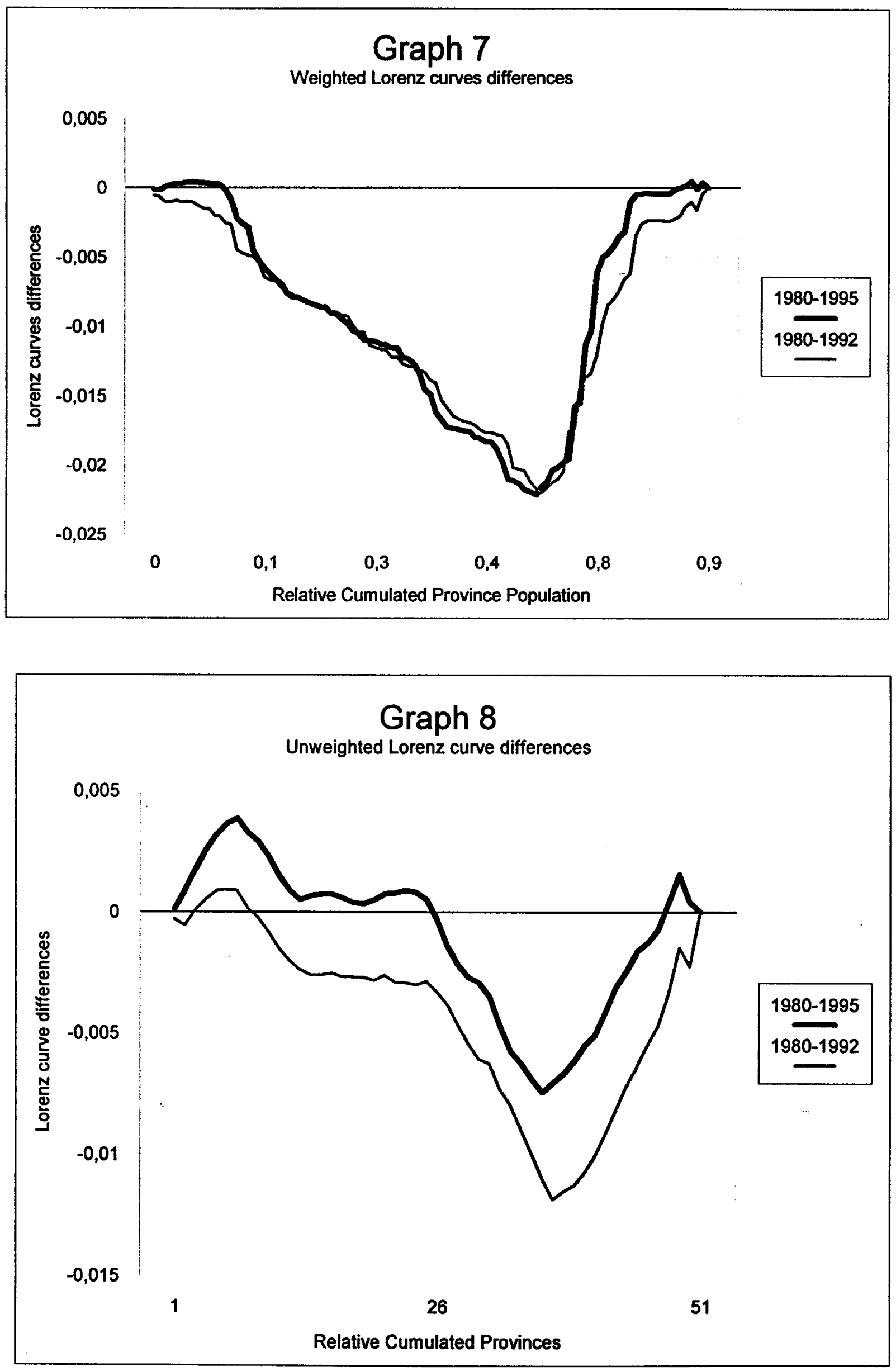




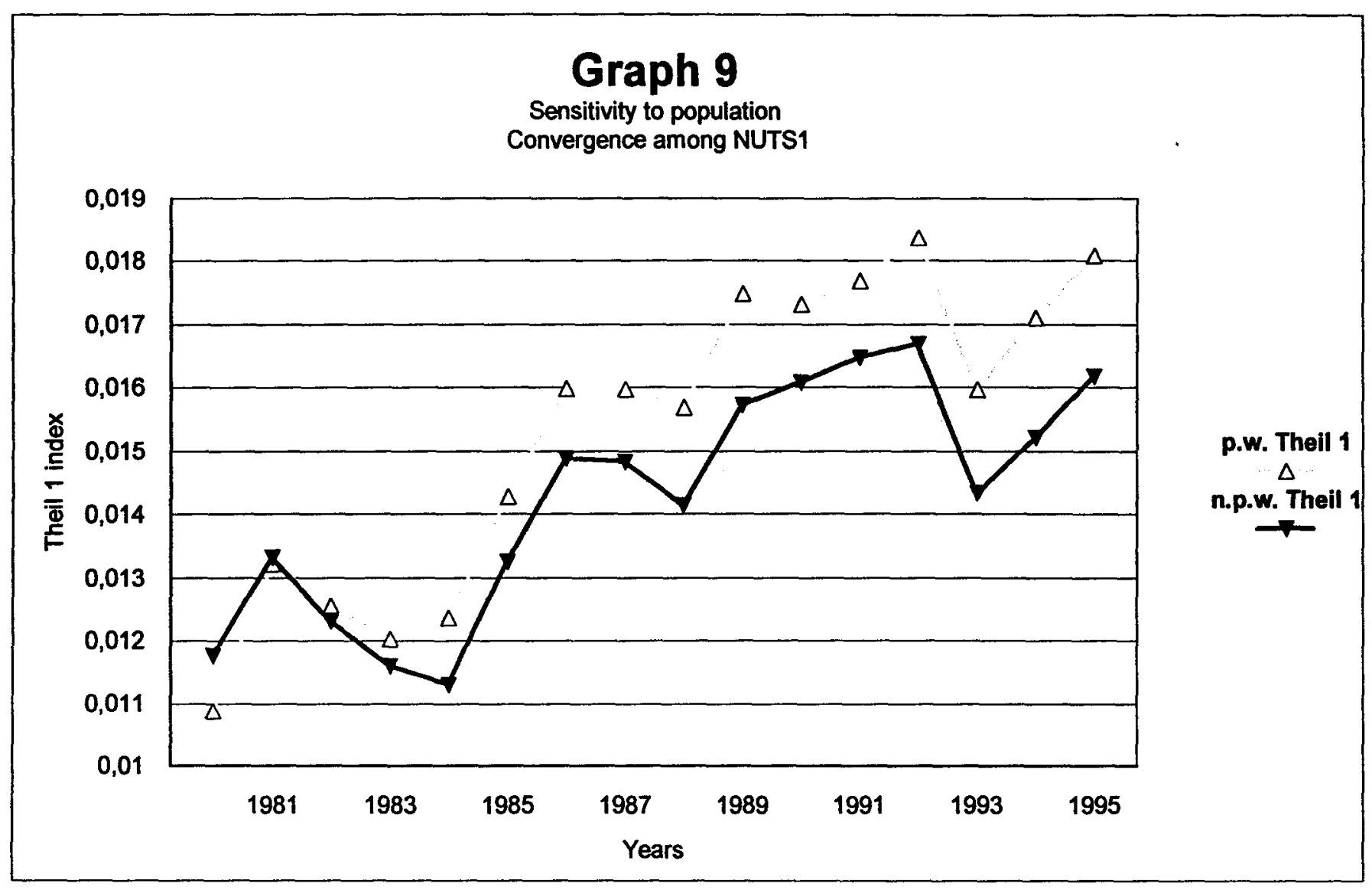



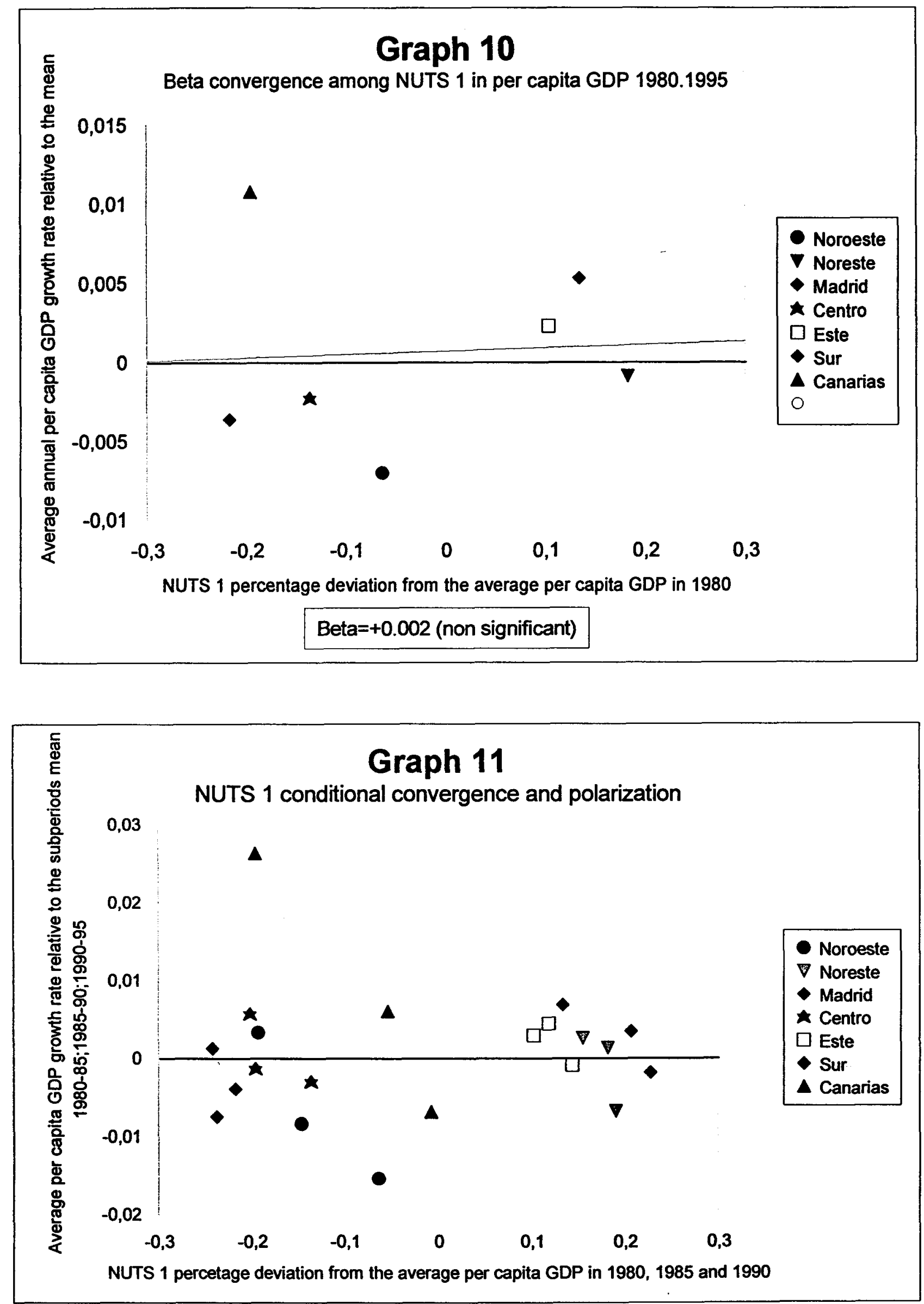


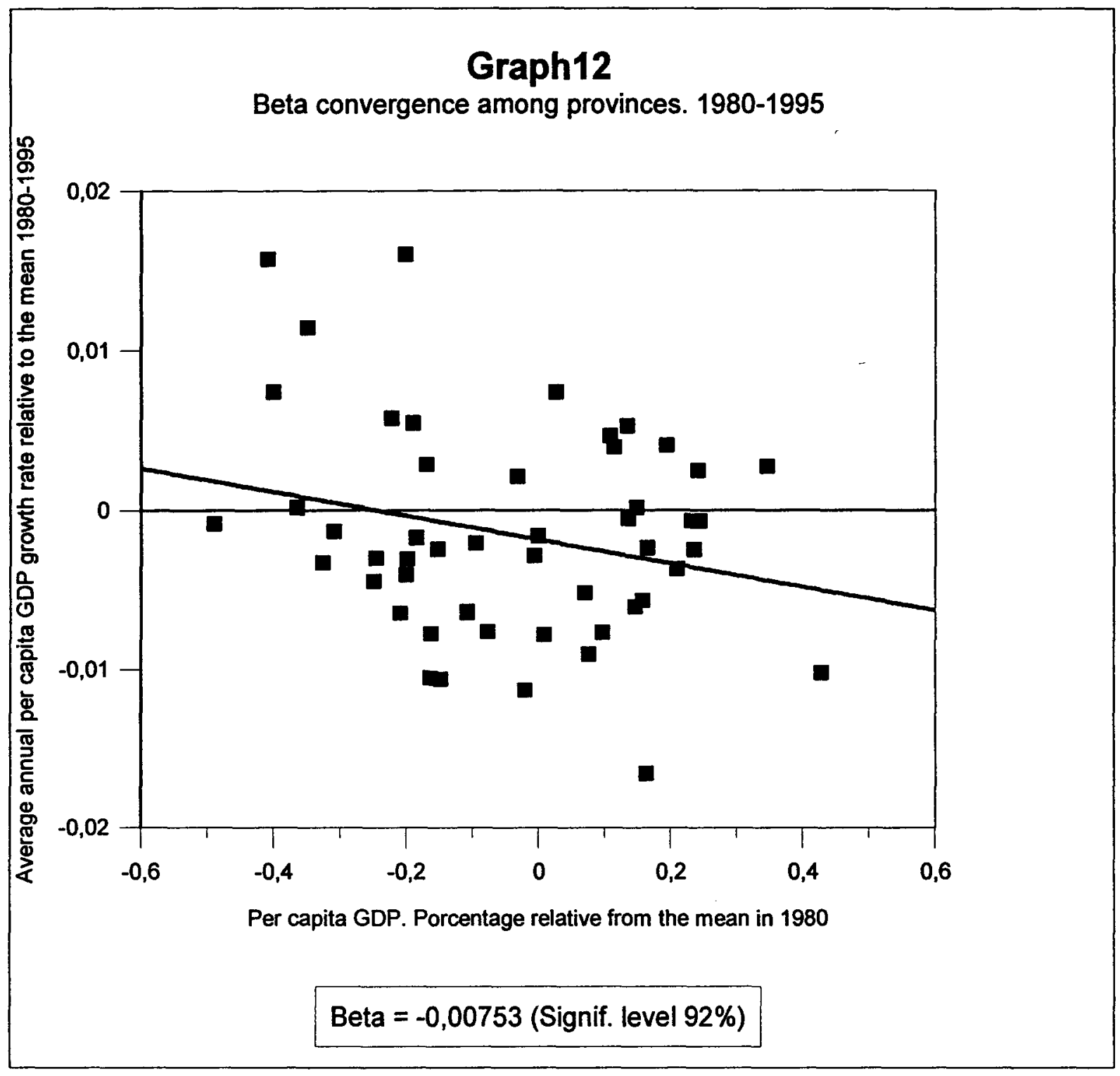




\section{REFERENCES:}

Atkinson, A.B. (1970): "On the Measurement of Inequality", Journal of Public Economics, 2, 244-263.

Barro, R. and X. Sala-i-Martin (1990): "Economic Growth and Convergence across the USA", NBER, WP3419.

Barro, R. and X. Sala-i-Martin (1992): "Convergence", Journal of Political Economy, 100, 223-251.

Cowell, F. (1985): "Multilevel Decomposition of Theil's Index of Inequality" The Review of Income and Wealth, 201-205.

Creedy, J. (1977): "The principle of transfers and the variance of logarithms", Oxford Bulletin of Economics. and Statistics, 39, 153-158.

Dasgupta, P., Sen, A. and D. Starret (1973): "Notes on the Measurement of Inequality", Journal of Economic Theory, 6, 180-187.

De la Fuente, A. (1996): "Convergencia y otras historias: economía regional desde una perspectiva neoclásica", Revista de Economía Aplicada, 4 (10), 5-64.

Mas, M., Maudos, J., Pérez, F. and E. Uriel (1994): "Disparidades regionales y convergencia en las Comunidades Autónomas" Revista de Economía Aplicada, 2 (4), 129-148.

Raymond, J. L. and B. García (1994): "Las disparidades en el PIB per capita entre Comunidades Autónomas y la hipótesis de convergencia", Papeles de Economía Española $59,37-58$. 
Rabadán I. and R. Salas (1996): "Convergencia y redistribución interterritorial en España: efecto de los impuestos directos, cotizaciones sociales y transferencias", Fundación BBV, Bilbao.

Shorrocks, A. (1980), "The Class of Additively Decomposable Inequality Measurements", Econometrica, 48, 613-625.

Shorrocks, A. (1983), "Ranking Income Distributions", Economica, 50, 3-17.

Wodon, Q.(1998), "Between Group Inequality and Targeted Transfers", The Rewiev of Income and Wealth, forthcoming. 


\section{Proof of Proposition 1}

If the inequality index is additive decomposable it can be written as:

$$
I\left(Y_{1, t}, Y_{2, t}, \ldots, Y_{n, t}\right)=I\left(\bar{Y}_{t}^{1} \Phi\left(n_{t}^{1}\right), \ldots, \bar{Y}_{t}^{K} \Phi\left(n_{t}^{K}\right)\right)+\sum_{k=1}^{K} w_{t}^{k}\left(\bar{Y}_{t}^{1}, n_{t}^{1}, \ldots, \bar{Y}_{t}^{K}, n_{t}^{K}\right) I\left(Y_{t}^{k}\right)
$$

and in turn,

$$
I\left(Y_{t}^{k}\right)=I\left(\bar{Y}_{t}^{k 1} \Phi\left(n_{t}^{k 1}\right), \ldots, \bar{Y}_{t}^{k S k} \Phi\left(n_{t}^{k S k}\right)\right)+\sum_{s \in k} w_{t}^{s}\left(\bar{Y}_{t}^{1}, n_{t}^{1}, \ldots, \bar{Y}_{t}^{S k}, n_{t}^{S k}\right) I\left(Y_{t}^{s}\right)
$$

so

$$
\begin{aligned}
& I\left(Y_{t}\right)=I\left(\bar{Y}_{t}^{1} \Phi\left(n_{t}^{1}\right), \ldots, \bar{Y}_{t}^{K} \Phi\left(n_{t}^{K}\right)\right)+\sum_{k=1}^{K} w_{t}^{k}\left(\bar{Y}_{t}^{1}, n_{t}^{1}, \ldots, \bar{Y}_{t}^{K}, n_{t}^{K}\right) I\left(\bar{Y}_{t}^{k 1} \Phi\left(n_{t}^{k 1}\right), \ldots, \bar{Y}_{t}^{k S k} \Phi\left(n_{t}^{k S k}\right)\right)+ \\
& \sum_{k=1}^{K} w_{t}^{k}\left(\bar{Y}_{t}^{1}, n_{t}^{1}, \ldots, \bar{Y}_{t}^{K}, n_{t}^{K}\right) \sum_{s \in k} w_{t}^{s}\left(\bar{Y}_{t}^{1}, n_{t}^{1}, \ldots, \vec{Y}_{t}^{S k}, n_{t}^{S k}\right) I\left(Y_{t}^{s}\right)
\end{aligned}
$$

As the index belongs to the General Entropy class the weights must take the form:

$$
w_{t}^{k}(.)=\frac{n_{t}^{k}}{n_{t}}\left(\frac{\bar{Y}_{1}^{k}}{\bar{Y}_{t}}\right)^{c}
$$

and we have

$$
\begin{aligned}
& I\left(Y_{t}\right)=I\left(\bar{Y}_{t}^{1} \Phi\left(n_{t}^{l}\right), \ldots, \bar{Y}_{t}^{K} \Phi\left(n_{t}^{K}\right)\right)+\sum_{k=1}^{K} \frac{n_{t}^{k}}{n_{t}}\left(\frac{\bar{Y}_{t}^{k}}{\bar{Y}_{t}}\right)^{c} I\left(\bar{Y}_{t}^{k 1} \Phi\left(n_{t}^{k 1}\right), \ldots, \bar{Y}_{t}^{k S k} \Phi\left(n_{t}^{k S k}\right)\right)+ \\
& \sum_{k=1}^{K} \frac{n_{t}^{k}}{n_{t}}\left(\frac{\bar{Y}_{t}^{k}}{\bar{Y}_{t}}\right)^{c} \sum_{s \in k} \frac{n_{t}^{s}}{n_{t}}\left(\frac{\bar{Y}_{t}^{s}}{\bar{Y}_{t}}\right)^{c} I\left(Y_{t}^{s}\right)
\end{aligned}
$$

so

$$
\begin{aligned}
& I\left(Y_{t}\right)=I\left(\bar{Y}_{t}^{1} \Phi\left(n_{t}^{1}\right), \ldots, \bar{Y}_{t}^{K} \Phi\left(n_{t}^{K}\right)\right)+\sum_{k=1}^{K} \frac{n_{t}^{k}}{n_{t}}\left(\frac{\bar{Y}_{t}^{k}}{\bar{Y}_{t}}\right)^{c} I\left(\bar{Y}_{t}^{k 1} \Phi\left(n_{t}^{k 1}\right), \ldots, \bar{Y}_{t}^{k S k} \Phi\left(n_{t}^{k S k}\right)\right)+ \\
& \sum_{s=1}^{S} \frac{n_{t}^{s}}{n_{t}}\left(\frac{\bar{Y}_{t}^{s}}{\bar{Y}_{t}}\right)^{c} I\left(Y_{t}^{s}\right)
\end{aligned}
$$

Then, the overall inequality index can be written as: 
$I\left(Y_{t}\right)=I\left(\bar{Y}_{t}^{1} \Phi\left(n_{t}^{1}\right), \ldots, \bar{Y}_{t}^{K} \Phi\left(n_{t}^{K}\right)\right)+\sum_{k=1}^{K} w_{t}^{k}(\cdot) I\left(\bar{Y}_{t}^{k 1} \Phi\left(n_{t}^{k 1}\right), \ldots, \bar{Y}_{t}^{k S k} \Phi\left(n_{t}^{k S k}\right)\right)+$ $\sum_{s=1}^{S} w_{1}^{s}(\cdot) I\left(Y_{1}^{s}\right)$

so the proposition is proved. The proof of Proposition 2 is analogous. 